\title{
The Bumpy Road towards mTOR Inhibition in Glioblastoma: Quo Vadis?
}

\author{
Kostas A. Papavassiliou (D) and Athanasios G. Papavassiliou *(D) \\ Department of Biological Chemistry, Medical School, National and Kapodistrian University of Athens, \\ 11527 Athens, Greece; konpapav@med.uoa.gr \\ * Correspondence: papavas@med.uoa.gr; Tel.: +30-210-746-2508
}

check for updates

Citation: Papavassiliou, K.A.;

Papavassiliou, A.G. The Bumpy Road towards mTOR Inhibition in

Glioblastoma: Quo Vadis?

Biomedicines 2021, 9, 1809. https://

doi.org/10.3390/biomedicines9121809

Academic Editor: Athanassios

P. Kyritsis

Received: 1 November 2021

Accepted: 30 November 2021

Published: 1 December 2021

Publisher's Note: MDPI stays neutral with regard to jurisdictional claims in published maps and institutional affiliations.

Copyright: (c) 2021 by the authors. Licensee MDPI, Basel, Switzerland. This article is an open access article distributed under the terms and conditions of the Creative Commons Attribution (CC BY) license (https:// creativecommons.org/licenses/by/ $4.0 /)$.

\begin{abstract}
Glioblastoma multiforme (GBM), a grade IV astrocytoma, is a lethal brain tumor with a poor prognosis. Despite recent advances in the molecular biology of GBM, neuro-oncologists have very limited treatment options available to improve the survival of GBM patients. A prominent signaling pathway implicated in GBM pathogenesis is that of the mechanistic target of rapamycin (mTOR). Attempts to target the mTOR pathway with first-generation mTOR inhibitors appeared promising in the preclinical stage; however, results have been disappointing in clinical trials, owing to the heterogeneous nature of GBM, escape mechanisms against treatment, the blood-brain barrier, drug-related toxicities, and the imperfect design of clinical trials, among others. The development of next-generation mTOR inhibitors and their current evaluation in clinical trials have sparked new hope to realize the clinical potential of mTOR inhibitors in GBM. Meanwhile, studies are continuously furthering our understanding of mTOR signaling dysregulation, its downstream effects, and interplay with other signaling pathways in GBM tumors. Therefore, it remains to be seen whether targeting mTOR in GBM will eventually prove to be fruitful or futile.
\end{abstract}

Keywords: glioblastoma multiforme; mTORC1; mTORC2; mTOR inhibitors; mTOR signaling

\section{Introduction}

Multiple oncogenic signaling pathways are known to be deregulated in glioblastoma multiforme (GBM), contributing to its pathogenesis. Among the signaling pathways that have been pharmacologically targeted in GBM is the mechanistic target of the rapamycin (mTOR) pathway. Although drugs modulating this pathway showed promising results in preclinical studies, their application in clinical trials proved to be disappointing, as a result of GBM heterogeneity, poor pharmacology, inherent and acquired resistance to mTOR inhibitors, and molecularly unselected cohorts of GBM patients in these clinical trials (Table 1) [1-3]. The failure of mTOR inhibitors to demonstrate clinical benefit until now does not mean that this therapeutic strategy should be discarded, but rather raises several important issues that need to be addressed in order to reach a verdict regarding the future of such agents in GBM management: (a) further research is required to uncover the intricacies of mTOR signaling in GBM, (b) preclinical studies assessing the therapeutic potential of mTOR inhibitors should represent, as close as possible, the conditions of the GBM tumor microenvironment, (c) rational subgroup selection of GBM patients in clinical trials based on the molecular profile of their tumors, and (d) combining mTOR inhibitors with other targeted agents. 
Table 1. Examples of novel mTOR inhibitors in GBM clinical trials.

\begin{tabular}{cc}
\hline mTOR Inhibitors & Clinical Trials \\
\hline Dual PI3K/mTOR inhibitors & \\
NVP-BEZ235 & Phase IIB (NCT02430363) \\
XL765 & Phase I/II (NCT01240460) \\
\hline mTORC1/mTORC2 inhibitors & \\
INK128 & Phase I (NCT02142803) \\
AZD8055 & Phase I (NCT01316809) \\
AZD2014 & Phase I (NCT02619864) \\
OSI-027 & Phase I (NCT00698243) \\
\hline
\end{tabular}

\section{Deconstructing mTOR Biology in GBM}

A deep understanding of the molecular circuitry of mTOR signaling in GBM is crucial in order to improve the clinical outcome of mTOR inhibitors. Research efforts have already provided a great amount of information on the role of the mTOR pathway in GBM pathogenesis [4,5]. Compared to mTORC1, less is known about mTORC2; however, the latter is emerging as a significant player in GBM, contributing to numerous oncogenic processes [6-8]. New data regarding the two complexes are helping to construct a more detailed picture of mTOR biology in GBM. For example, a recent study revealed that GBM stem cells (GSCs) can activate the mTOR pathway in GBM-associated microglia, resulting in an immunosuppressive microenvironment that promotes GBM growth [9]. As the authors state, these findings suggest that mTOR inhibitors could also act on microglia to suppress their tumor-promoting effects. Another recent study discovered that both mTORC1 and mTORC2, acting downstream of oncogenic epidermal growth factor receptor (EGFR) signaling, are cooperatively involved in the epigenetic regulation of GBM progression. More specifically, mTORC1 was shown to increase the protein levels of the enhancer of zeste homolog 2 (EZH2), a histone methyltransferase that constitutes the catalytic subunit of polycomb repressive complex 2 (PRC2) and catalyzes the tri-methylation of histone $\mathrm{H} 3$ at lysine 27 (H3K27me3), whereas mTORC2 functions to upregulate the intracellular concentration of S-adenosylmethionine (SAM), the substrate for histone methylation. The coordinated activity of both mTOR complexes was demonstrated to promote H3K27me3 and drive GBM growth in vitro and in vivo [10]. Furthermore, mTORC2 functions as an epigenetic regulator of iron metabolism to promote GBM cell survival [11]. Knowledge of the complexity of mTOR signaling in GBM, including regulatory mechanisms and molecular cross-talks with other pathways, is increasingly growing. For example, there is crosstalk between PI3K/mTOR and MEK/ERK signaling that drives the self-renewal maintenance and tumorigenicity of GBM stem cells (GSCs) [12]. Evidence also suggests an interaction between mTORC2 and Hippo signaling that leads to the promotion of GBM growth and invasiveness $[13,14]$. Concerning regulatory mechanisms, mTORC2 is implicated in a feed-forward loop, involving Akt, heat-shock transcription factor 1 (HSF1), human antigen $\mathrm{R}(\mathrm{HuR})$, and Rictor, which enhances mTORC2 activity and GBM growth [15]. All these aspects of mTOR signaling have opened new avenues for research in GBM biology and have provided opportunities for targeting the mTOR pathway through different strategies.

\section{Novel mTOR Inhibitors}

First-generation mTOR inhibitors include rapamycin and chemical compounds derived from rapamycin (rapalogs) that act to specifically block the mTORC1 complex via binding to FK506 binding protein 12 (FKBP12) [16]. Several aspects related to the molecular architecture of mTOR signaling have been found to be responsible for its incomplete suppression by first-generation mTOR inhibitors and the failure of the latter in GBM clinical trials. These include disinhibition of mTORC1-mediated negative feedback loops by rapamycin, which results in the activation of protein kinase $\mathrm{B}(\mathrm{Akt})$, eukaryotic translation initiation factor $4 \mathrm{E}$ (eIF4E), and mitogen-activated protein kinase (MAPK) survival pathways [17-19]; the presence of mTORC1 kinase-dependent activity that is resistant to 
rapamycin [20]; and the insensitivity of mTORC2 to acute treatment with rapamycin, which allows Akt to be activated [21,22]. The limited capacity of first-generation mTOR inhibitors led to the development of second-generation mTOR inhibitors that bind to the ATP-binding pocket in the mTOR kinase domain of both mTORC1 and mTORC2 and repress their activity. Second-generation mTOR inhibitors include dual phosphatidylinositol-3 kinase (PI3K)/mTOR inhibitors (e.g., PI-103, GNE-477, NVP-BEZ235, BGT226, XL765, SF-1126, and WJD008) and mTORC1/2 inhibitors (e.g., Torin1, Torin2, PP242, PP30, Ku-0063794, WAY-600, WYE-687, WYE-354, INK128, AZD8055, AZD2014, and OSI-027) [23]. Many second-generation mTOR inhibitors have now progressed to GBM clinical trials, and the results of these trials are eagerly awaited. The inevitable emergence of resistance mechanisms to first- and second-generation mTOR inhibitors has fueled research efforts to develop third-generation mTOR inhibitors: bivalent compounds that link rapamycin to second-generation mTOR inhibitors [24]. RapaLink-1, a third-generation mTOR inhibitor capable of crossing the blood-brain barrier, was shown to be more potent in in vitro and in vivo GBM models than earlier generation mTOR inhibitors [25]. Another promising class of mTOR inhibitors that is still in the early phase of drug development is selective mTORC2 inhibitors. The rationale behind the development of such compounds is that the selective blockade of only mTORC2 will not interfere with mTORC1-dependent negative feedback loops and, thus, may be more effective in inhibiting tumor growth [26]. A study identified CID613034 and its analog JR-AB2-011, small molecule inhibitors that are highly selective for mTORC2, which displayed potent anti-tumor effects in GBM cell lines and xenografts, respectively [27]. These novel mTOR inhibitors bring mTOR inhibition in GBM back to the spotlight and offer hope for future GBM clinical trials (Table 2).

Table 2. Next-generation mTOR inhibitors.

\begin{tabular}{|c|c|}
\hline mTOR Inhibitors & Mechanism of Action \\
\hline $\begin{array}{c}\frac{\text { Second-generation mTOR inhibitors }}{\bullet \text { Dual PI3K/mTOR inhibitors }} \\
\text { (PI-103, GNE-477, NVP-BEZ235, BGT226, } \\
\text { XL765, SF-1126, and WJD008) }\end{array}$ & $\begin{array}{l}\text { Bind to ATP-binding pocket of both mTOR and } \\
\text { PI3K }\end{array}$ \\
\hline $\begin{array}{c}\text { Second-generation mTOR inhibitors } \\
\qquad \bullet \text { mTORC1/mTORC2 inhibitors } \\
\text { (Torin1, Torin2, PP242, PP30, Ku-0063794, } \\
\text { WAY-600, WYE-687, WYE-354, INK128, } \\
\text { AZD8055, AZD2014, and OSI-027) }\end{array}$ & $\begin{array}{l}\text { Bind to ATP-binding pocket in the mTOR kinase } \\
\text { domain of both mTORC1 and mTORC2 }\end{array}$ \\
\hline$\frac{\text { Third-generation mTOR inhibitors }}{\text { RapaLink-1, RapaLink-2 }}$ & $\begin{array}{l}\text { Bind to FKBP12 and ATP-binding pocket of } \\
\text { mTORC1 (pharmacophores connected via a linker) }\end{array}$ \\
\hline
\end{tabular}

\section{Biologically Relevant Preclinical GBM Studies Accessing mTOR Inhibitors}

Ideally, preclinical studies should use in vitro and in vivo models that reflect, as much as possible, the biology of GBM tumors in order to evaluate the effect of targeted drugs and proceed to clinical trials. A recent study that highlights the significance of this concept used the mTORC1/2 inhibitors Torin2, INK-128, and NVP-BEZ235 to investigate their effects on GBM metabolism. Interestingly, the authors discovered that under hypoxic and nutrientpoor conditions, mTORC $1 / 2$ inhibitors-much like rapamycin and rapalogs-protected GBM cells by increasing their tolerance to nutrient and oxygen deprivation [28]. GBM tumors are primarily characterized by a nutrient-deficient hypoxic microenvironment; hence, mTOR inhibitors should be used cautiously in GBM patients. This study also hints at the following ideas: (a) combining mTOR inhibitors with other therapies that block the pro-survival effects induced by the former or (b) developing selective mTOR inhibitors that only promote GBM growth inhibition without eliciting a protective response against hypoxia and nutrient deficiency. 


\section{Designing Appropriate GBM Clinical Trials for mTOR Inhibitors}

A major drawback of the clinical trials that failed so far is the inclusion of GBM patients without molecularly defining their tumors in terms of mTOR signaling activity. If the clinical outcomes of mTOR inhibitors for GBM are to be improved, the identification and incorporation of predictive biomarkers that can define patients who are most likely to show a response to mTOR inhibitors in clinical trials is of utmost importance. A paradigm of such a molecularly informed clinical trial is that of the novel German $\mathrm{N}^{2} \mathrm{M}^{2}$ trial, which evaluates molecularly matched targeted therapies for newly diagnosed unmethylated isocitrate dehydrogenase (IDH) wild-type GBM patients. Specifically, one of the goals of this trial is to molecularly analyze patient tumor samples, identify and select those patients whose tumors exhibit activated mTOR (mTOR phosphorylated at Ser2448), and subsequently administer temsirolimus along with standard radiotherapy to this particular patient subgroup [29]. Nevertheless, the fact that patients' brain tumors harbor particular molecular alterations that are used as potential predictive biomarkers does not automatically translate into a sensitivity to drugs targeting these alterations because GBM tumors present remarkable spatial and temporal heterogeneity.

Phase 0 and window of opportunity clinical trials are also a step forward toward accelerating the development of effective mTOR inhibitors and other drugs for GBM; however, it is not without its challenges, given the obstacle of delivering drugs to the central nervous system due to the blood-brain barrier and the difficulty of acquiring brain tumor biopsies [30]. Such studies, if their clinical protocols are standardized, can rapidly assess the pharmacokinetic and pharmacodynamic properties of mTOR-targeting compounds on a patient's tumors. Therefore, if a compound demonstrates poor pharmacology early on-for example, not being able to penetrate the blood-brain barrier-its further development can immediately be halted.

Obtaining brain tumor tissues from GBM patients at relevant time points during treatment in clinical trials to assess the adequacy of target inhibition is difficult, but it can provide vital information about the biological effects of targeted drugs on patient-derived GBM cells as well as the molecular mechanisms of resistance to targeted therapy. Despite the challenges, a Phase I trial successfully applied this strategy to evaluate the antitumor activity of rapamycin in patients with recurrent phosphatase and tensin homolog (PTEN)deficient GBM [31]. Similar trials in the future will help pave the way toward developing effective mTOR inhibitors.

Another innovation that will assist in the improvement of the clinical outcome of mTOR inhibitors is the development of noninvasive methods that are able to directly or indirectly assess whether these drugs are, in fact, hitting their intended target. For example, a recent study identified 2-hydroxyglutarate (2HG), via magnetic resonance spectroscopy, as a metabolic biomarker of IDH-mutant GBM response to the dual PI3K/mTOR inhibitor XL765. Additionally, the decreased 2HG levels detected after treatment with XL765 were correlated with improved survival in a GBM mouse model [32]. Further preclinical and clinical studies are required to validate this biomarker.

\section{6. mTOR Inhibition in Combination with Other Targeted Therapies}

GBM is not a single-pathway disease. Either due to intrinsic multiple oncogenic pathways or the activation of cross-talk and feedback mechanisms in response to targeted therapy, monotherapies are not sufficient to inhibit GBM tumor growth. Unfortunately, much like the disappointing results of the GBM clinical trials using only mTOR inhibitors, the combination of mTOR inhibition with standard chemoradiation yielded poor results [3] However, preclinical data indicate a synergistic effect of mTOR inhibition with TMZ that may be based on crosstalk between the mTOR pathway and the TMZ-mediated cell death pathway $[33,34]$. Deciphering the molecular details of this crosstalk may reveal why this therapeutic combination did not translate into an improved clinical outcome. Hence, combining mTOR inhibitors with different drugs targeting other signaling pathways is a much more effective therapeutic approach against GBM, as it improves responses 
to therapy, overcomes therapy resistance, and addresses GBM heterogeneity. Research on polytherapies for GBM has proposed various combinations, such as pairing mTOR inhibitors with mitogen-activated protein kinase (MEK), cyclin-dependent kinase 4 and 6 (CDK4/6), mouse double minute 2 homolog (MDM2), signal transducer and activator of transcription 3 (STAT3), or growth hormone-releasing hormone (GHRH) inhibitors [35-39]. These and other combination therapies warrant further evaluation to validate which ones are worth positioning for clinical development.

\section{Conclusions}

mTOR represents a hallmark-signaling pathway in GBM and there is a strong rationale for its therapeutic targeting in this aggressive brain tumor. The unexpected results from recent clinical trials of $\mathrm{mTOR}$ inhibitors were met with disappointment from the neurooncology community. However, rather than abandoning the road for mTOR inhibition in GBM, physicians and researchers in the field should view this outcome as an opportunity to learn, improve, and subsequently pivot to the most promising path forward. As outlined here, it becomes evident that in order to unleash the full potential of mTOR inhibitors in GBM, an integrated approach is required, encompassing improvements in clinical trial design and preclinical studies, a deep understanding of mTOR biology in GBM, the development of more selective mTOR inhibitors, and the identification of the most effective combination therapies based on mTOR inhibitors. Only when all the above are considered and addressed will we arrive at a solid conclusion with respect to the future of mTOR inhibitors in GBM therapy. Results from each front will help direct our next steps on this challenging road of mTOR inhibition in GBM. Most likely, mTOR inhibitors will prove to be effective for a subset of GBM patients with a relevant molecular profile and will eventually be springboarded into the neuro-oncology clinic.

Author Contributions: Writing—original draft preparation, K.A.P.; writing-review and editing, A.G.P. All authors have read and agreed to the published version of the manuscript.

Funding: This research received no external funding.

Conflicts of Interest: The authors declare no conflict of interest.

\section{References}

1. Chang, S.M.; Wen, P.; Cloughesy, T.; Greenberg, H.; Schiff, D.; Conrad, C.; Fink, K.; Robins, H.I.; De Angelis, L.; Raizer, J.; et al. Phase II study of CCI-779 in patients with recurrent glioblastoma multiforme. Investig. New Drugs 2005, 23, 357-361. [CrossRef] [PubMed]

2. Galanis, E.; Buckner, J.C.; Maurer, M.J.; Kreisberg, J.I.; Ballman, K.; Boni, J.; Peralba, J.M.; Jenkins, R.B.; Dakhil, S.R.; Morton, R.F.; et al. Phase II trial of temsirolimus (CCI-779) in recurrent glioblastoma multiforme: A North Central Cancer Treatment Group Study. J. Clin. Oncol. 2005, 23, 5294-5304. [CrossRef]

3. Chinnaiyan, P.; Won, M.; Wen, P.Y.; Rojiani, A.M.; Werner-Wasik, M.; Shih, H.A.; Ashby, L.S.; Michael Yu, H.H.; Stieber, V.W.; Malone, S.C.; et al. A randomized phase II study of everolimus in combination with chemoradiation in newly diagnosed glioblastoma: Results of NRG Oncology RTOG 0913. Neuro-Oncology 2018, 20, 666-673. [CrossRef]

4. Jhanwar-Uniyal, M.; Gillick, J.L.; Neil, J.; Tobias, M.; Thwing, Z.E.; Murali, R. Distinct signaling mechanisms of mTORC1 and mTORC2 in glioblastoma multiforme: A tale of two complexes. Adv. Biol. Regul. 2015, 57, 64-74. [CrossRef]

5. Jhanwar-Uniyal, M.; Wainwright, J.V.; Mohan, A.L.; Tobias, M.E.; Murali, R.; Gandhi, C.D.; Schmidt, M.H. Diverse signaling mechanisms of mTOR complexes: mTORC1 and mTORC2 in forming a formidable relationship. Adv. Biol. Regul. 2019, 72, 51-62. [CrossRef] [PubMed]

6. Chantaravisoot, N.; Wongkongkathep, P.; Loo, J.A.; Mischel, P.S.; Tamanoi, F. Significance of filamin A in mTORC2 function in glioblastoma. Mol. Cancer 2015, 14, 127. [CrossRef] [PubMed]

7. Gu, Y.; Albuquerque, C.P.; Braas, D.; Zhang, W.; Villa, G.R.; Bi, J.; Ikegami, S.; Masui, K.; Gini, B.; Yang, H.; et al. mTORC2 Regulates Amino Acid Metabolism in Cancer by Phosphorylation of the Cystine-Glutamate Antiporter xCT. Mol. Cell 2017, 67, 128-138.e7. [CrossRef] [PubMed]

8. Masui, K.; Cavenee, W.K.; Mischel, P.S. mTORC2 and Metabolic Reprogramming in GBM: At the Interface of Genetics and Environment. Brain Pathol. 2015, 25, 755-759. [CrossRef]

9. Dumas, A.A.; Pomella, N.; Rosser, G.; Guglielmi, L.; Vinel, C.; Millner, T.O.; Rees, J.; Aley, N.; Sheer, D.; Wei, J.; et al. Microglia promote glioblastoma via mTOR-mediated immunosuppression of the tumour microenvironment. EMBO J. 2020, 39, e103790. [CrossRef] [PubMed] 
10. Harachi, M.; Masui, K.; Honda, H.; Muragaki, Y.; Kawamata, T.; Cavenee, W.K.; Mischel, P.S.; Shibata, N. Dual Regulation of Histone Methylation by mTOR Complexes Controls Glioblastoma Tumor Cell Growth via EZH2 and SAM. Mol. Cancer Res. 2020, 18, 1142-1152. [CrossRef]

11. Masui, K.; Harachi, M.; Ikegami, S.; Yang, H.; Onizuka, H.; Yong, W.H.; Cloughesy, T.F.; Muragaki, Y.; Kawamata, T.; Arai, N.; et al. mTORC2 links growth factor signaling with epigenetic regulation of iron metabolism in glioblastoma. J. Biol. Chem. 2019, 294, 19740-19751. [CrossRef]

12. Sunayama, J.; Matsuda, K.; Sato, A.; Tachibana, K.; Suzuki, K.; Narita, Y.; Shibui, S.; Sakurada, K.; Kayama, T.; Tomiyama, A.; et al. Crosstalk between the PI3K/mTOR and MEK/ERK pathways involved in the maintenance of self-renewal and tumorigenicity of glioblastoma stem-like cells. Stem Cells 2010, 28, 1930-1939. [CrossRef]

13. Holmes, B.; Benavides-Serrato, A.; Saunders, J.T.; Kumar, S.; Nishimura, R.N.; Gera, J. mTORC2-mediated direct phosphorylation regulates YAP activity promoting glioblastoma growth and invasive characteristics. Neoplasia 2021, 23, 951-965. [CrossRef]

14. Artinian, N.; Cloninger, C.; Holmes, B.; Benavides-Serrato, A.; Bashir, T.; Gera, J. Phosphorylation of the Hippo Pathway Component AMOTL2 by the mTORC2 Kinase Promotes YAP Signaling, Resulting in Enhanced Glioblastoma Growth and Invasiveness. J. Biol. Chem. 2015, 290, 19387-19401. [CrossRef]

15. Holmes, B.; Benavides-Serrato, A.; Freeman, R.S.; Landon, K.A.; Bashir, T.; Nishimura, R.N.; Gera, J. mTORC2/AKT/HSF1/HuR constitute a feed-forward loop regulating Rictor expression and tumor growth in glioblastoma. Oncogene 2018, 37, 732-743. [CrossRef]

16. Choi, J.; Chen, J.; Schreiber, S.L.; Clardy, J. Structure of the FKBP12-rapamycin complex interacting with the binding domain of human FRAP. Science 1996, 273, 239-242. [CrossRef]

17. O'Reilly, K.E.; Rojo, F.; She, Q.B.; Solit, D.; Mills, G.B.; Smith, D.; Lane, H.; Hofmann, F.; Hicklin, D.J.; Ludwig, D.L.; et al. mTOR inhibition induces upstream receptor tyrosine kinase signaling and activates Akt. Cancer Res. 2006, 66, 1500-1508. [CrossRef]

18. Sun, S.Y.; Rosenberg, L.M.; Wang, X.; Zhou, Z.; Yue, P.; Fu, H.; Khuri, F.R. Activation of Akt and eIF4E survival pathways by rapamycin-mediated mammalian target of rapamycin inhibition. Cancer Res. 2005, 65, 7052-7058. [CrossRef]

19. Carracedo, A.; Ma, L.; Teruya-Feldstein, J.; Rojo, F.; Salmena, L.; Alimonti, A.; Egia, A.; Sasaki, A.T.; Thomas, G.; Kozma, S.C.; et al. Inhibition of mTORC1 leads to MAPK pathway activation through a PI3K-dependent feedback loop in human cancer. J. Clin. Investig. 2008, 118, 3065-3074. [CrossRef]

20. Thoreen, C.C.; Kang, S.A.; Chang, J.W.; Liu, Q.; Zhang, J.; Gao, Y.; Reichling, L.J.; Sim, T.; Sabatini, D.M.; Gray, N.S. An ATPcompetitive mammalian target of rapamycin inhibitor reveals rapamycin-resistant functions of mTORC1. J. Biol. Chem. 2009, 284, 8023-8032. [CrossRef]

21. Jacinto, E.; Loewith, R.; Schmidt, A.; Lin, S.; Rüegg, M.A.; Hall, A.; Hall, M.N. Mammalian TOR complex 2 controls the actin cytoskeleton and is rapamycin insensitive. Nat. Cell Biol. 2004, 6, 1122-1128. [CrossRef]

22. Sarbassov, D.D.; Guertin, D.A.; Ali, S.M.; Sabatini, D.M. Phosphorylation and regulation of Akt/PKB by the rictor-mTOR complex. Science 2005, 307, 1098-1101. [CrossRef]

23. Zhou, H.Y.; Huang, S.L. Current development of the second generation of mTOR inhibitors as anticancer agents. Chin. J. Cancer 2012, 31, 8-18. [CrossRef]

24. Rodrik-Outmezguine, V.S.; Okaniwa, M.; Yao, Z.; Novotny, C.J.; McWhirter, C.; Banaji, A.; Won, H.; Wong, W.; Berger, M.; de Stanchina, E.; et al. Overcoming mTOR resistance mutations with a new-generation mTOR inhibitor. Nature 2016, 534, $272-276$. [CrossRef]

25. Fan, Q.; Aksoy, O.; Wong, R.A.; Ilkhanizadeh, S.; Novotny, C.J.; Gustafson, W.C.; Truong, A.Y.; Cayanan, G.; Simonds, E.F.; Haas-Kogan, D.; et al. A Kinase Inhibitor Targeted to mTORC1 Drives Regression in Glioblastoma. Cancer Cell 2017, 31, 424-435. [CrossRef]

26. Murray, E.R.; Cameron, A. Towards specific inhibition of mTORC2. Aging 2017, 9, 2461-2462. [CrossRef]

27. Benavides-Serrato, A.; Lee, J.; Holmes, B.; Landon, K.A.; Bashir, T.; Jung, M.E.; Lichtenstein, A.; Gera, J. Specific blockade of Rictor-mTOR association inhibits mTORC2 activity and is cytotoxic in glioblastoma. PLoS ONE 2017, 12, e0176599. [CrossRef]

28. Heinzen, D.; Divé, I.; Lorenz, N.I.; Luger, A.L.; Steinbach, J.P.; Ronellenfitsch, M.W. Second Generation mTOR Inhibitors as a Double-Edged Sword in Malignant Glioma Treatment. Int. J. Mol. Sci. 2019, 20, 4474. [CrossRef]

29. Wick, W.; Dettmer, S.; Berberich, A.; Kessler, T.; Karapanagiotou-Schenkel, I.; Wick, A.; Winkler, F.; Pfaff, E.; Brors, B.; Debus, J.; et al. N2M2 (NOA-20) phase I/II trial of molecularly matched targeted therapies plus radiotherapy in patients with newly diagnosed non-MGMT hypermethylated glioblastoma. Neuro-Oncology 2019, 21, 95-105. [CrossRef]

30. Vogelbaum, M.A.; Krivosheya, D.; Borghei-Razavi, H.; Sanai, N.; Weller, M.; Wick, W.; Soffietti, R.; Reardon, D.A.; Aghi, M.K.; Galanis, E.; et al. Phase 0 and window of opportunity clinical trial design in neuro-oncology: A RANO review. Neuro-Oncology 2020, 22, 1568-1579. [CrossRef]

31. Cloughesy, T.F.; Yoshimoto, K.; Nghiemphu, P.; Brown, K.; Dang, J.; Zhu, S.; Hsueh, T.; Chen, Y.; Wang, W.; Youngkin, D.; et al. Antitumor activity of rapamycin in a Phase I trial for patients with recurrent PTEN-deficient glioblastoma. PLoS Med. 2008, 5, e8. [CrossRef]

32. Batsios, G.; Viswanath, P.; Subramani, E.; Najac, C.; Gillespie, A.M.; Santos, R.D.; Molloy, A.R.; Pieper, R.O.; Ronen, S.M. $\mathrm{PI} 3 \mathrm{~K} / \mathrm{mTOR}$ inhibition of IDH1 mutant glioma leads to reduced 2HG production that is associated with increased survival. Sci. Rep. 2019, 9, 10521. [CrossRef] 
33. Zając, A.; Sumorek-Wiadro, J.; Langner, E.; Wertel, I.; Maciejczyk, A.; Pawlikowska-Pawlega, B.; Pawelec, J.; Wasiak, M.; HułasStasiak, M.; Bądziul, D.; et al. Involvement of PI3K Pathway in Glioma Cell Resistance to Temozolomide Treatment. Int. J. Mol. Sci. 2021, 22, 5155. [CrossRef]

34. Luchman, H.A.; Stechishin, O.D.; Nguyen, S.A.; Lun, X.Q.; Cairncross, J.G.; Weiss, S. Dual mTORC1/2 blockade inhibits glioblastoma brain tumor initiating cells in vitro and in vivo and synergizes with temozolomide to increase orthotopic xenograft survival. Clin. Cancer Res. 2014, 20, 5756-5767. [CrossRef]

35. Schreck, K.C.; Allen, A.N.; Wang, J.; Pratilas, C.A. Combination MEK and mTOR inhibitor therapy is active in models of glioblastoma. Neuro-Oncol. Adv. 2020, 2, vdaa138. [CrossRef]

36. Olmez, I.; Brenneman, B.; Xiao, A.; Serbulea, V.; Benamar, M.; Zhang, Y.; Manigat, L.; Abbas, T.; Lee, J.; Nakano, I.; et al. Combined CDK4/ 6 and mTOR Inhibition Is Synergistic against Glioblastoma via Multiple Mechanisms. Clin. Cancer Res. 2017, 23, 6958-6968. [CrossRef]

37. Daniele, S.; Costa, B.; Zappelli, E.; Da Pozzo, E.; Sestito, S.; Nesi, G.; Campiglia, P.; Marinelli, L.; Novellino, E.; Rapposelli, S.; et al. Combined inhibition of AKT/mTOR and MDM2 enhances Glioblastoma Multiforme cell apoptosis and differentiation of cancer stem cells. Sci. Rep. 2015, 5, 9956. [CrossRef]

38. Miyata, H.; Ashizawa, T.; Iizuka, A.; Kondou, R.; Nonomura, C.; Sugino, T.; Urakami, K.; Asai, A.; Hayashi, N.; Mitsuya, K.; et al. Combination of a STAT3 Inhibitor and an mTOR Inhibitor against a Temozolomide-resistant Glioblastoma Cell Line. Cancer Genom. Proteom. 2017, 14, 83-91. [CrossRef]

39. Kiaris, H.; Chatzistamou, I.; Papavassiliou, A.G.; Schally, A.V. Growth hormone-releasing hormone: Not only a neurohormone. Trends Endocrinol. Metab. 2011, 22, 311-317. [CrossRef] 